GLASNIK MATEMATIČKI

Vol. 40(60)(2005), $339-345$

\title{
A LOCAL TO GLOBAL SELECTION THEOREM FOR SIMPLEX-VALUED FUNCTIONS
}

\author{
IVAN IVANŠIĆ AND LEONARD R. Rubin \\ University of Zagreb, Croatia and University of Oklahoma, USA
}

\begin{abstract}
Suppose we are given a function $\sigma: X \rightarrow K$ where $X$ is a paracompact space and $K$ is a simplicial complex, and an open cover $\left\{U_{\alpha} \mid \alpha \in \Gamma\right\}$ of $X$, so that for each $\alpha \in \Gamma, f_{\alpha}: U_{\alpha} \rightarrow|K|$ is a map that is a selection of $\sigma$ on its domain. We shall prove that there is a map $f: X \rightarrow|K|$ which is a selection of $\sigma$. We shall also show that under certain conditions on such a set of maps or on the complex $K$, there exists a $\sigma: X \rightarrow K$ with the property that each $f_{\alpha}$ is a selection of $\sigma$ on its domain and that there is a selection $f: X \rightarrow|K|$ of $\sigma$. The term selection, as used herein, will always refer to a map $f$, i.e., continuous function, having the property that $f(x) \in \sigma(x)$ for each $x$ in the domain.
\end{abstract}

\section{Introduction}

The purpose of this paper is to prove certain selection theorems of the type "local to global" where the target is a polyhedron. We will apply an enhanced version from [6] of a method due to E. Michael [5] to pass from local selections to global ones.

Throughout this paper, map will mean continuous function and if $K$ is a simplicial complex, then its polyhedron $|K|$ will always have the weak topology induced by the triangulation $K$. As in [1] and [5], paracompact spaces are assumed to be Hausdorff.

Let us recall that if $K$ is a simplicial complex and $f: X \rightarrow|K|$ and $g: X \rightarrow|K|$ are functions, then $f$ is said to be contiguous to $g$ if for each $x \in X$ there exists a simplex $\tau \in K$ such that $f(x), g(x) \in \tau$; one says that $g$ is a $K$-modification of $f$ if for each $x \in X$, whenever $f(x) \in \tau \in K$, then

2000 Mathematics Subject Classification. 54C65, 54C05, 54E20.

Key words and phrases. Contiguous functions, continuous function, discrete collection, infinite simplex, $K$-modification, locally finite-dimensional complex, paracompact, polyhedron, principal simplex, selection, simplex, simplicial complex. 
$g(x) \in \tau$. When $f$ is contiguous to $g$, it need not be true that either one of them is a $K$-modification of the other. Also contiguity is not an equivalence relation although it is reflexive and symmetric.

Let $\sigma: X \rightarrow K$ be a function where $X$ is a space and $K$ is a simplicial complex. If $\left\{U_{\alpha} \mid \alpha \in \Gamma\right\}$ is an open cover of $X$ and for each $\alpha \in \Gamma, f_{\alpha}: U_{\alpha} \rightarrow$ $|K|$ is a map, then the statement that $\left\{f_{\alpha} \mid \alpha \in \Gamma\right\}$ is a selection of $\sigma$ means that for each $\alpha \in \Gamma$ and $x \in U_{\alpha}, f_{\alpha}(x) \in \sigma(x)$. This is a generalization of the usual notion of a selection because if $\Gamma=\{\alpha\}$ is singleton and $U_{\alpha}=X$, then $f_{\alpha}$ is a selection in the usual sense.

If we consider a family $\left\{f_{\alpha} \mid \alpha \in \Gamma\right\}$ of maps $f_{\alpha}$ of subsets $U_{\alpha}$ of $X$ to $|K|$ (e.g., as above), one may say that the maps $f_{\alpha}$ are "pointwise contiguous in pairs" if for each $x \in X$ and $\alpha, \beta \in \Gamma$ such that $x \in U_{\alpha} \cap U_{\beta}, f_{\alpha}(x)$ and $f_{\beta}(x)$ lie in a simplex of $K$.

We now state the main results of this paper, the first of which strengthens Theorem 1.2 of [2] by removing the requirement that the space $X$ be hereditarily normal.

TheOrem 1.1. Let $X$ be a paracompact space, $K$ a simplicial complex, and $\sigma: X \rightarrow K$ a function. Suppose that $\left\{U_{\alpha} \mid \alpha \in \Gamma\right\}$ is an open cover of $X$ and $\left\{f_{\alpha}: U_{\alpha} \rightarrow|K| \mid \alpha \in \Gamma\right\}$ is a selection of $\sigma$. Then there exists a selection $f: X \rightarrow|K|$ of $\sigma$.

The term "infinite simplex" is probably not in common use.

Definition 1.2. Let $K$ be a simplicial complex and $\left\{\tau_{i} \mid i \in \mathbb{N}\right\}$ a collection of simplexes of $K$ such that for each $i \in \mathbb{N}, \operatorname{dim} \tau_{i}=i-1$ and $\tau_{i}$ is a face of $\tau_{i+1}$. Then we shall refer to $\left\{\tau_{i} \mid i \in \mathbb{N}\right\}$ as an infinite simplex of $K$.

A simplicial complex $K$ contains an infinite simplex if and only if there is a full subcomplex $L$ of $K$ which has a countably infinite set of vertices. Although infinite simplexes are not simplicial complexes, the concept is useful as follows. If we are given a collection $\left\{f_{\alpha} \mid \alpha \in \Gamma\right\}$ of maps $f_{\alpha}$ of subsets $U_{\alpha}$ of $X=\{0\}$ that is pointwise contiguous in pairs to the polyhedron $|K|$ of the full complex $K(\mathbb{N})$ whose vertex set is $\mathbb{N}$, there need not be a $\sigma: X \rightarrow K(\mathbb{N})$ such that this collection is a selection for $\sigma$. For example, fix an infinite simplex $\left\{\tau_{i} \mid i \in \mathbb{N}\right\}$ in $K(\mathbb{N})$, and for each $i \in \mathbb{N}$, let $f_{i}(0)$ be the barycenter of $\tau_{i}$.

The statement that a simplicial complex $K$ contains no infinite simplexes is equivalent to the statement that each simplex of $K$ is contained in a principal simplex, that is, one which is contained in no other simplex of $K$.

TheOREm 1.3. Let $X$ be a paracompact space, $K$ a simplicial complex, and $\left\{U_{\alpha} \mid \alpha \in \Gamma\right\}$ an open cover of $X$. Suppose that for each $\alpha \in \Gamma$, there exists a map $f_{\alpha}: U_{\alpha} \rightarrow|K|$ such that if $\alpha, \beta \in \Gamma$, then one of the maps $f_{\alpha} \mid U_{\alpha} \cap U_{\beta}$ and $f_{\beta} \mid U_{\alpha} \cap U_{\beta}$ is a $K$-modification of the other. If either,

(A) : $K$ contains no infinite simplex or, 
(B) : $\left\{U_{\alpha} \mid \alpha \in \Gamma\right\}$ is a point-finite open cover of $X$,

then there exist,

1. $\sigma: X \rightarrow K$ such that $\left\{f_{\alpha} \mid \alpha \in \Gamma\right\}$ is a selection of $\sigma$, and

2. a map $f: X \rightarrow|K|$ such that $f$ is a selection of $\sigma$.

The following lemma is an easy consequence of the definitions.

Lemma 1.4. Let $K$ be a simplicial complex, $X$ a space, $\sigma: X \rightarrow K a$ function, $\left\{U_{\alpha} \mid \alpha \in \Gamma\right\}$ an open cover of $X$, and for each $\alpha \in \Gamma, f_{\alpha}: U_{\alpha} \rightarrow|K|$ a map. If $\left\{f_{\alpha} \mid \alpha \in \Gamma\right\}$ and $f: X \rightarrow|K|$ are selections of $\sigma$, then $f \mid U_{\alpha}$ is contiguous to $f_{\alpha}$ for each $\alpha \in \Gamma$.

Lemma 1.4 shows that in both Theorem 1.1 and Theorem 1.3, for each $\alpha \in \Gamma, f \mid U_{\alpha}$ is contiguous to $f_{\alpha}$. It is worth noting (for example, Lemma $4^{\prime}$ of [4]) that this implies that $f \mid U_{\alpha} \simeq f_{\alpha}$.

\section{LEMmAS}

Let us state Lemma 1.1 of [2] which, as indicated in the latter, follows from the proof of Lemma 4 of [4].

LEMMA 2.1. Let $X$ be a normal space and $K$ a simplicial complex. Let $A \subset X$ be a closed set and $V, U \subset X$ open sets such that $A \subset V \subset \bar{V} \subset U$. If $h: U \rightarrow|K|$ and $g: V \rightarrow|K|$ are maps such that $h \mid V$ is contiguous to $g$, then there exists a map $k: U \rightarrow|K|$ such that:

1. $k$ is contiguous to $h$,

2. $k|A=g| A$,

3. $k|(U \backslash V)=h|(U \backslash V)$, and

4. if $x \in V$ and $h(x), g(x) \in \sigma \in K$, then $k(x) \in \sigma$.

LEMMA 2.2. Let $K$ be a simplicial complex that has no infinite simplexes and $\left\{x_{\alpha} \mid \alpha \in \Gamma\right\}$ be a nonempty subset of $|K|$ such that for each $\alpha, \beta \in \Gamma$, at least one of the following is true:

1. if $x_{\alpha} \in \tau \in K$, then $x_{\beta} \in \tau$, or

2. if $x_{\beta} \in \tau \in K$, then $x_{\alpha} \in \tau$.

Then there exists a principal simplex $\sigma$ of $K$ such that $\left\{x_{\alpha} \mid \alpha \in \Gamma\right\} \subset \sigma$.

Proof. It is sufficient to find a simplex $\sigma$ of $K$ such that $x_{\alpha} \in \sigma$ for all $\alpha \in \Gamma$. Fix $\alpha_{1} \in \Gamma$ and let $\sigma_{1}$ be the simplex of $K$ such that $x_{\alpha_{1}} \in \operatorname{int} \sigma_{1}$. Define $\Gamma_{1}=\left\{\gamma \in \Gamma \mid x_{\gamma} \in \sigma_{1}\right\}$. If $\Gamma_{1}=\Gamma$, then put $\sigma=\sigma_{1}$, and we are done. Otherwise, choose $\alpha_{2} \in \Gamma \backslash \Gamma_{1}$. Let $\sigma_{2}$ be the simplex of $K$ such that $x_{\alpha_{2}} \in \operatorname{int} \sigma_{2}$. Since $x_{\alpha_{2}} \notin \sigma_{1}$, then (1) and (2) of the hypothesis show that we must have $x_{\alpha_{1}} \in \sigma_{2}$. But then $\sigma_{2}$ intersects the interior of $\sigma_{1}$, showing that $\sigma_{1}$ is a face of $\sigma_{2}$. Put $\Gamma_{2}=\left\{\gamma \in \Gamma \mid x_{\gamma} \in \sigma_{2}\right\}$. Clearly, $\Gamma_{1} \subset \Gamma_{2}$. If $\Gamma_{2}=\Gamma$, 
then put $\sigma=\sigma_{2}$, and we are done. If one continues this process, it will end after a finite number, say $n$, of steps since $K$ has no infinite simplexes. This means that $\Gamma_{n}=\Gamma$. Designate $\sigma=\sigma_{n}$. Our proof is complete.

In case the complex $K$ in Lemma 2.2 contains infinite simplexes, then the following result can be useful. Its proof can be modelled on the preceding one.

Lemma 2.3. Let $K$ be a simplicial complex and $\Sigma$ be a nonempty finite subset of $|K|$ such that for each $x, y \in \Sigma$, at least one of the following is true:

1. if $x \in \tau \in K$, then $y \in \tau$, or

2. if $y \in \tau \in K$, then $x \in \tau$.

Then there exists a simplex $\sigma$ of $K$ such that $\Sigma \subset \sigma$.

Recall that a simplicial complex $K$ is called locally finite-dimensional if for each vertex $v$ of $K$ there exists a nonnegative integer $n$ such that $\operatorname{dim} \tau \leq n$ for each simplex $\tau$ of $K$ having $v$ as a vertex.

Corollary 2.4. If $K$ is a locally finite-dimensional simplicial complex, then $K$ has no infinite simplexes.

The next statement follows from Corollary 2.4 and (A) of Theorem 1.3.

Corollary 2.5. Let $X$ be a paracompact space, $K$ a locally finitedimensional simplicial complex, and $\left\{U_{\alpha} \mid \alpha \in \Gamma\right\}$ an open cover of $X$. Suppose that for each $\alpha \in \Gamma$, there exists a map $f_{\alpha}: U_{\alpha} \rightarrow|K|$ such that if $\alpha$, $\beta \in \Gamma$, then one of the maps $f_{\alpha} \mid U_{\alpha} \cap U_{\beta}$ and $f_{\beta} \mid U_{\alpha} \cap U_{\beta}$ is a K-modification of the other. Then there exist,

1. $\sigma: X \rightarrow K$ such that $\left\{f_{\alpha} \mid \alpha \in \Gamma\right\}$ is a selection of $\sigma$, and

2. a map $f: X \rightarrow|K|$ such that $f$ is a selection of $\sigma$.

\section{Proofs of Theorems}

Recall that a collection $\mathcal{K}$ of subsets of a space is called discrete if each point of the space has a neighborhood that intersects at most one element of the collection. An application of Theorem 1.1.13 of [1] shows that this is true if and only if the collection of the closures of the elements of $\mathcal{K}$ is discrete.

In [6] we observed that Theorem 3.6(a) of E. Michael [5] could be improved by applying Michael's Proposition 3.3 in his proof of that theorem. This was stated as Lemma 1 in [6]. Here it is in a slightly different form (taking into account the citation from [1] mentioned in the preceding paragraph).

Proposition 3.1. Let $X$ be a paracompact space and $\mathcal{G}$ a collection of subsets of $X$. Suppose that the following are true:

1. $\mathcal{G}$ contains an open cover of $X$, 
2. if $U \in \mathcal{G}$ and $W$ is open in $U$, then $W \in \mathcal{G}$,

3. if $U, Q$ are open elements of $\mathcal{G}$, then $U \cup Q \in \mathcal{G}$, and

4. if $\mathcal{K} \subset \mathcal{G}$ is a discrete collection of open subsets of $X$, then $\bigcup \mathcal{K} \in \mathcal{G}$.

Then the entire space $X$ is in $\mathcal{G}$.

We now give our proof of Theorem 1.1.

Proof. Let $\mathcal{G}$ be the collection of all open subsets $G$ of $X$ such that there exists an open neighborhood $U$ of $\bar{G}$ and a selection $h: U \rightarrow|K|$ of $\sigma \mid U$. Our proof will be complete if we show that $X \in \mathcal{G}$, and we shall apply Proposition 3.1 to do this. To obtain (1) of Proposition 3.1, just apply the shrinking theorem for normal spaces to the open cover $\left\{U_{\alpha} \mid \alpha \in \Gamma\right\}$. Item (2) is obviously so.

To obtain (3) of Proposition 3.1, suppose that $U_{0}$ and $Q_{0}$ are in $\mathcal{G}$. Choose open sets $U, Q$ of $X$ such that $\bar{U}_{0} \subset U, \bar{Q}_{0} \subset Q$ and maps $h: U \rightarrow|K|$, $g: Q \rightarrow|K|$ so that the pair $\{h, g\}$ is a selection of the restriction of $\sigma$ to $U \cup Q$. Also let $U_{1}, U_{2}, Q_{1}, Q_{2}$ be sets open in $X$ such that, $\bar{U}_{0} \subset U_{1} \subset \bar{U}_{1} \subset$ $U_{2} \subset \bar{U}_{2} \subset U$, and $\bar{Q}_{0} \subset Q_{1} \subset \bar{Q}_{1} \subset Q_{2} \subset \bar{Q}_{2} \subset Q$.

Put $A=\bar{U}_{1} \cap \bar{Q}_{1}$ and $V=U_{2} \cap Q_{2}$. Apply Lemma 2.1 to the preceding data and let $k: U \rightarrow|K|$ be as indicated there. Define a map $l: \bar{U}_{1} \cup \bar{Q}_{1} \rightarrow|K|$ by,

$$
l(x)= \begin{cases}g(x) & \text { if } x \in \bar{Q}_{1}, \\ k(x) & \text { if } x \in \bar{U}_{1} .\end{cases}
$$

An application of (2) of Lemma 2.1 shows that $l$ is a well-defined map. Let us show that $l$ is a selection of $\sigma \mid \bar{U}_{1} \cup \bar{Q}_{1}$. Since $x \in \bar{Q}_{1}$ implies that $l(x)=g(x) \in \sigma(x)$, then we only have to consider $x \in \bar{U}_{1} \backslash \bar{Q}_{1}$. Hence $l(x)=k(x)$ and $x \in U$. There are two cases.

Case 1. $x \in U \backslash V$. By (3) of Lemma 2.1, $k(x)=h(x) \in \sigma(x)$.

Case 2. $x \in V=U_{2} \cap Q_{2} \subset U \cap Q$. Then because of the selection assumption, $h(x), g(x) \in \sigma(x)$, so by (4) of Lemma 2.1, $l(x)=k(x) \in \sigma(x)$.

Of course this implies that $l\left|U_{1} \cup Q_{1}: U_{1} \cup Q_{1} \rightarrow\right| K \mid$ is a selection of $\sigma \mid U_{1} \cup Q_{1}$. Surely, $U_{1} \cup Q_{1}$ is an open neighborhood of $\overline{U_{0} \cup Q_{0}}=\bar{U}_{0} \cup \bar{Q}_{0}$, so we have demonstrated that (3) holds true.

At last, (4) of Proposition 3.1 is obtained by a simple application of the fact that $X$ is collectionwise normal (see, e.g., Theorem 5.1.18 of [1]).

Here is our proof of Theorem 1.3.

Proof. Consider first (A). Let $x \in X$; put $\Gamma_{x}=\left\{\alpha \in \Gamma \mid x \in U_{\alpha}\right\}$. Suppose that $\alpha, \beta \in \Gamma_{x}$. Since one of the maps $f_{\alpha} \mid U_{\alpha} \cap U_{\beta}$ and $f_{\beta} \mid U_{\alpha} \cap U_{\beta}$ is a $K$-modification of the other, then at least one of the conditions (1), (2) of Lemma 2.2 is true. In order to obtain $\sigma: X \rightarrow K$, apply Lemma 2.2 to $\Gamma_{x}$, 
obtaining a principal simplex $\sigma(x) \in K$ such that $x_{\alpha}=f_{\alpha}(x) \in \sigma(x)$ for all $\alpha \in \Gamma_{x}$. Certainly $\left\{f_{\alpha} \mid \alpha \in \Gamma\right\}$ is a selection of $\sigma: X \rightarrow K$. The existence of a map $f: X \rightarrow|K|$ such that $f$ is a selection of $\sigma$ follows from Theorem 1.1.

A proof of (B) can be done by the same technique as the preceding, using Lemma 2.3 in place of Lemma 2.2.

Let us close the paper with a Proposition showing that the infinite simplex hypothesis in part (A) of Theorem 1.3 cannot be dismissed.

Proposition 3.2. Part (A) of Theorem 1.3 may fail to be true if $K$ contains infinite simplexes.

Proof. As before, let $K(\mathbb{N})$ be the full complex whose vertex set is $\mathbb{N}$. Define $X$ to be $\mathbb{N}$ with the discrete topology. Then $X$ is a paracompact space. For each $n \in \mathbb{N}$, let $\tau_{n}$ be the simplex of $K(\mathbb{N})$ whose vertex set is $\{k \in \mathbb{N} \mid k \leq n\}$, and put $b_{n}$ equal the barycenter of $\tau_{n}$.

For each $n \in \mathbb{N}$, let $U_{n}=\{1, n\}$ and $f_{n}: U_{n} \rightarrow|K(\mathbb{N})|$ be given by $f_{n}(t)=b_{n}$ for all $t \in U_{n}$. Surely, $\left\{U_{n} \mid n \in \mathbb{N}\right\}$ is an open cover of $X$ and for each $n \in \mathbb{N}, f_{n}: U_{n} \rightarrow|K(\mathbb{N})|$ is a map. Also, if $m, n \in \mathbb{N}$, then $U_{m} \cap U_{n}=\{1\}$. If $m \leq n$ and $f_{n}(1)=b_{n}$ lies in a simplex $\tau$ of $K(\mathbb{N})$, then $\tau_{n}$ is a face of $\tau$ and $f_{m}(1)=b_{m}$ lies in a face of $\tau_{n}$, so it lies in a face of $\tau$. Hence, one of $f_{m} \mid U_{m} \cap U_{n}$ and $f_{n} \mid U_{m} \cap U_{n}$ is a $K(\mathbb{N})$-modification of the other. But there is no $\sigma: X \rightarrow K(\mathbb{N})$ such that $\left\{f_{n} \mid n \in \mathbb{N}\right\}$ is a selection of $\sigma$ because no simplex from $K(\mathbb{N})$ contains $\left\{f_{n}(1) \mid n \in \mathbb{N}\right\}$.

\section{ACKNOWLEDGEMENTS.}

The authors are most grateful to the referee who made a careful and thoughtful reading of this paper and whose suggestions are now incorporated into it.

\section{REFERENCES}

1. R. Engelking, General Topology PWN-Polish Scientific Publishers, Warsaw, 1977.

2. I. Ivanšić and L. Rubin, A selection theorem for simplex-valued maps, Glas. Mat. Ser. III 39(59) (2004), 331-333.

3. S. Mardešić, Extension dimension of inverse limits, Glas. Mat. Ser. III 35(55) (2000), 339-354.

4. S. Mardešić, Extension dimension of inverse limits. Correction of a proof., Glas. Mat. Ser. III 39(59) (2004), 335-337.

5. E. Michael, Local properties of topological spaces, Duke Mat. J. 21 (1954), 163-171.

6. L. Rubin, Relative collaring, Proc. Amer. Math. Soc. 55 (1976), 181-184. 
I. Ivanšić

Department of Mathematics

University of Zagreb

Unska 3, P.O. Box 148

10001 Zagreb

Croatia

E-mail: ivan.ivansic@fer.hr

L.R. Rubin

Department of Mathematics

University of Oklahoma

Norman, Oklahoma 73019

USA

E-mail: lrubin@ou.edu

Received: 3.2.2005.

Revised: 31.3.2005. 